\title{
NOTAS SOBRE LA OBRA CIENTÍFICA DE COSERIU (15)
}

\author{
José POLO \\ Universidad Autónoma de Madrid \\ jose.polo@uam.es \\ II \\ ARQUITECTURA DE LA LENGUA \\ 4: «parada y fonda» aminorada
}

0

Las presentes notas han sido redactadas bajo el proyecto Lingüistica de Eugenio Coseriu y lingüistica coseriana (Ministerio de Ciencia y Tecnología, FFI2008-04605/FILO).

$\mathrm{A} / 1$

El anterior microsistema de la serie presente consistió en hacer desfilar lecturas varias de Coseriu en las cuales dicho estudioso había hallado referencias a los niveles socioculturales de la lengua (eje diastrático) y a las modalidades lingüísticas según la situación comunicativa o estilos de lengua (eje diafásico). Ello ha sido entre los números 16/2002 y el 21/2007 (salvo el 17/2003, volumen especial en el que no se insertó esta serie); así, pues, cinco entregas dedicadas a rastrear pistas bibliográficas en las lecturas coserianas para dos de los ejes dentro de la «arquitectura de la lengua» Redondeado tal segmento bibliográfico-metodológico, ha llegado el momento de hacer entrar en juego los diversos trabajos de Coseriu en los cuales, con una u otra terminología, atisbó, planteó o desarrolló lo relativo a esa doble perspectiva de los niveles y estilos de lengua. Advierto, sin embargo, que ya en la segunda entrega de la serie (ELUA, 8/1992, págs. 201-210) puse en el centro del espacio lector el núcleo de esta clase de perspectivas cuando me referí a un estudio fundacional de Leiv Flydal y a diversos trabajos de Coseriu en los que, haciéndose cargo del planteamiento del lingüista noruego, ampliaba el espectro añadiendo una cuarta coordenada: la del eje diafásico. No obstante dicho anticipo conceptual, llegará un momento, dentro de la serie, en que me veré obligado a colocar, o recolocar, en su sitio los trabajos aludidos del lingüista rumano-uruguayo para que dicha piedra angular sirva de entrada a los propios desarrollos ulteriores, a su repercusión dentro y fuera del mundo hispánico (a partir de una referencia en una conocida obra sociolingüística de Brigitte Schlieben-Lange, presencia o ausencia de tales planteamientos coserianos en la nueva etapa de la Escuela de Praga, en los ámbitos hispanohablante, germanohablante, etc., entre sus discípulos o fuera de tal espacio de cercanía metodológica). 


\section{$\mathrm{B} / 2$}

Pero, como especie de interludio entre el microsistema anterior y el que enseguida vendrá (origen múltiple, en su propia obra, del pensamiento coseriano al respecto), bueno será, en esta parte del recorrido, volver a presentar la ficha del aludido decisivo trabajo del estudioso noruego Leiv FLYDAL, a saber: «Remarques sur certains rapports entre le style et l'état de langue», en Norsk Tidsskrift for Sprogvidenskap, XVI/1952, págs. 241-258. Y lo hago no por mera repetición mecánica, sino porque, justamente, en el primer párrafo de su estudio reconoce (además, claro está, de al omnipresente Ferdinand de Saussure con su sincronía/diacronía y aledaños) dos fuentes que le sirvieron de estímulo para replantear una serie de cuestiones que desembocaron en el sólido y fructífero esquema tripartito (luego cuatripartito en el asedio llevado a cabo por Coseriu). He aquí sus palabras (pág. 241, inicio del artículo):

C'est une étude de M. R.-L. Wagner, En marge d'un problème de syntaxe (L'ordre de phrase sujet+verbe) (Mélanges Hœpffner, Paris, 1949 [Mélanges de philologie romane et de littérature médiévale offerts a Ernest Hoepffner par ses élèves et ses amis, París, 1949 ; reimpreso por Slaykine Reprints, Ginebra, 1974]), qui a donné lieu aux considérations qui vont suivre. Nous avons essayé d'arriver à une représentation d'ensemble, réduite à sa plus simple expression, des rapports existant entre certains aspects du style et la langue. Nous renvoyons à Hans Vogt, Språksystem og språkutvikling (NTS 1947, Bind 14 [ Norsk Tidsskrift for Sprogvidenskap, XIV/1947, págs. 293-304]) notamment pour ce que nous avons appelé l'integration momentanée à une structure donnée d'un système partiel antérieur. Cette formule, qui permet de conserver intacte, en face des abondantes survivances qui caractérisent certaines langues, la distinction de principe établie par Saussure entre les plans synchronique et diachronique, nous avons tâché de l'élargir en l'appliquant à d'autres perspectives que celle temporelle et de lui donner ainsi une validité plus générale.

3

Y, paralelamente, el primer párrafo del trabajo de Robert Léon Wagner y el último en su parte inicial contienen los principios que han alumbrado la aplicación metodológica proyectada hacia el asunto consignado en el subtítulo. No voy a citar nada, por razones comprensibles, del trabajo de Hans Vogt, pero sí los mencionados segmentos textuales del artículo del estudioso francés (págs. 53 y 61 ; los separo mediante pleca doble negrita):

D'après le schéma saussurien, la linguistique historique a charge de définir et d'expliquer le rapport de deux faits de langue successifs, sans relier ceux-ci, pour autant, aux systèmes dont ils font partie. On peut figurer toute explication de ce genre para le symbole AllB, où les deux lettres représenten les faits de langue, et la flèche le procès, au long de la durée, qui conduit de l'un à l'autre. Commode, à la rigueur, lorsqu'on travaille sur des formes, ce schéma devient une gêne dès qu'on aborde, en syntaxe, les faits de structure. Dégageant en effet deux éléments de la langue, les isolant de leur contexte, il suggère, non sans risques, que l'évolution de A en B était en quelque sorte préfigurée, A contenant en soi, à l'état virtuel, tous les traits qui caractérisent B par rapport à lui ; d'une façon implicite il introduit, en histoire, un finalisme dont les linguistes ne sauraient trop se défier.ll Au début de cette note, j'exprimais une réserve sur la validité du schéma AllB en syntaxe. L'exemple tiré de la succession des ordres possibles entre sujet (nominal ou pronominal) et verbe illustre, mieux que tout autre, combien le procès lui-même de $\mathrm{A}$ à $\mathrm{B}$ est complexe, puisqu'y jouent $\grave{a}$ la 
fois et la résistance de certains types de phrase et l'influence que la construction d'autres types numériquement bien représentés exerce sur eux et encore la volonté consciente chez quelques écrivains ou l'instinct obscur chez d'autres d'obvier à des confusions gênantes en éliminant certaines constructions. On peut élargir encore les réflexions auxquelles incite cette histoire et trouver là un nouveau moyen de définir les rapports du style et de la langue. Si par fait de structure on entende l'obligation pour le sujet parlant de se soumettre à un ordre de phrase infrangible, et par [par] fait de style un choix laissé libre entre deux ordres, les termes extrêmes du procés en question font figure dans la langue de fait de structure.

$\mathrm{C} / 4$

En la entrega número 8 de esta serie (ELUA, 14/2000, págs. 291-299), hallándome en la zona del concepto de «dialectos verticales/dialectos sociales», que había hecho arrancar de Vicente García de Diego, texto de 1926) y que desarrollé, haciendo desfilar a estudiosos que lo mencionaban, etc., en las entregas aparecidas en los volúmenes 12/1998 (págs. 327-334), 13/1999 (págs, 297-305), 14/2000 (págs. 291-299) y 15/2001 (págs. 381-385), citaba yo, del penúltimo tomo señalado, un trabajo de Francisco Abad Nebot de 1989 (págs. 294-295) y, para lo que ahora nos interesa, una determinada afirmación (pág. 295), a saber (los corchetes, instalados ya en esa época, salvo el tercero, de ahora, son todos míos):

No obstante estas anticipaciones de García de Diego y cualquiera [cualesquiera] otras que se quieran buscar incluso en él mismo [véase la entrega número 6 de esta serie], la verdad es que la ciencia lingüística no vendría a hacerse cargo de un modo sistemático de la dialectalidad interna vertical del idioma [a partir de García de Diego: «dialectos internos verticales»] hasta la década de los años sesenta [afirmación discutible: ya Coseriu, en 1958, en congreso, había cimentado la perspectiva de lo sociocultural en el lenguaje: véase la entrega número 2, §5-A]. Entre los autores de habla hispana se adelantó ligeramente José Pedro Rona, en un a modo de manifiesto programático que también es necesario conocer: el que llamó Algunos [palabra que no pertenece al título de la obra] aspectos de la dialectología hispanoamericana [omito nota 5; en su momento estudiaré, dentro de esta serie, las relaciones entre maestro, Coseriu, y discípulo, Rona, en torno al asunto que nos ocupa].

\section{5}

Atenuaré el sintagma, interpolado por mí, «afirmación discutible», pues el aludido congreso en el que participó Coseriu no tuvo actas y fue bastantes años después («Los conceptos de "dialecto", "nivel" y "estilo de lengua" y el sentido propio de la dialectología», en Lingüistica Española Actual, III-1/1981, págs. 1-32), como sabemos, cuando pudimos conocer ese texto, aunque, como se irá viendo a partir de la siguiente entrega, en la propia obra coseriana de sus comienzos científicos ya merodeaba, o se instalaba con diferente terminología y en forma más o menos diluida en comparación con su precisa y definitiva formulación ulterior, la idea de lo social-estilístico (ejes diastrático y diafásico). Pero, en fin, cual acabo de señalar, será hacia el final de esta serie cuando podamos contar con elementos de juicio sólidos para matizar o no la en principio defendible, en cuanto idea general más bien de connotación «fática», afirmación del profesor Abad Nebot. Dejémosla estar... 
Pero, sin salirnos de este espacio, cambiemos de tercio. En carta de fecha 21 de marzo del 2001, el colega David L. Gold (a quien públicamente doy las gracias por la sistemática y rápida atención que siempre presta, en muy oportunos comentarios epistolares, a mis trabajos), refiriéndose a la página 295 , sintagma «afirmación discutible» interpolado por mí en la cita atrás, 4 , reproducida, me escribe (los dos primeros corchetes son míos):

No cabe duda [en cuanto al sintagma «afirmación discutible» con respecto a lo expresado por Abad Nebot]. De hecho, ya hace muchos años (¿desde Bloomfield?[¿en la «edición» de 1914 de su obra más conocida o en la de 1933?]) que se dice en círculos lingüísticos de habla inglesa que «all language [incluso la lengua literaria o la lengua estándar] is dialect». Como Coseriu es, desgraciadamente, poco conocido en dichos círculos (casi nunca lo veo citado en publicaciones inglesas), no puede ser él el que introdujo dicha idea en esos círculos y, por lo tanto, supongo que Coseriu no fue el primero en concebirlas. IIDialecto vertical: el concepto es conocidísimo en círculos lingüísticos de habla inglesa (desde hace muchos años) $=$ ingl. sociolect $\mathrm{y}$ vertical dialect.

7

Unos días después de recibir su carta con diversos comentarios (relacionados con las varias separatas de mis trabajos últimos enviadas), contesté a las preguntas que se me hacían atinentes a esos materiales remitidos y, naturalmente, me hice cargo también del comentario acabado de reproducir (sin entrar en el hecho de la presencia o ausencia científica de Coseriu en el ámbito anglohablante, pues ello requiere no ya un comentario, sino una larga explanación historiográfica que nos lleva forzosamente al planteamiento de diversas cuestiones relacionadas con la metodología científica y con la sociología, y hasta con la ecología, de la práctica de la ciencia). Esta fue mi contestación al respecto:

En los próximos números de esta serie aparecerán fichados multitud de trabajos en inglés, francés, alemán, etc., en los que se habla de todo esto (áparecerán también ideas de Coseriu muy anteriores a 1958). Entonces quedará relativamente claro lo básico de la cronología, etc., de aparición de estos y otros conceptos.

8

En efecto: todo el microsistema inmediatamente anterior (las lecturas de Coseriu con referencia a los ejes diastrático y diafásico) es parte del anunciado desfile bibliográfico de trabajos en lenguas varias, así como los que en las próximas entregas irán apareciendo. No obstante, la pregunta sobre quién o dónde se empleó por primera vez lo de dialecto vertical y sociolecto, en qué ámbito lingüístico, supone una investigación muy amplia, ajena al sentido de la presente, la cual, sin embargo, dentro de su limitado propósito, dejará expedita, o casi, una ruta con la que se podrá operar a la hora de contrastar los datos que en ella se presentan con los de otros mundos (geográficos, metodológicos, etc.). En todo caso, resulta muy expresiva la presencia, desde 1926 en adelante, en García de Diego de lo de dialectos internos verticales y dialectos sociales; supone ello una llamativa e informativa concentración «sociolingüística» que, sospecho, probablemente no se haya dado con un grado tal de explicitud conceptual y terminológica en ningún otro espacio de la investigación en torno al lenguaje (cuando menos, en el ámbito hispánico). Puestos, sin embargo, a buscar alguna fuente en dicho estudioso, téngase en cuenta su voluminosa obra Lingüistica general y 
española (CSIC, Madrid, 1951), pues, aunque la fecha de publicación es muy posterior a 1926, aparecen citados y, sin duda, aprovechados estudios anteriores a 1926 y otros a 1951 (la bibliografía al final del volumen ya nos dice algo en esta línea). Pero prestemos atención, además, en la pertenencia, «a su manera», a la rama filológica del Centro de Estudios Históricos (1910-1938) de nuestro autor y el servicio que los fondos bibliotecarios de dicha institución prestó a todos ellos (Menéndez Pidal, Américo Castro, Tomás Navarro Tomás, etcétera) y, por lo tanto, igualmente, a don Vicente García de Diego, que también, al igual que muchos otros, ha podido beneficiarse de la lectura o consulta de obras de lingüística presentes en la Real Academia Española y en El Ateneo de Madrid. En fin, intento, con estas «divagaciones», señalar que no me cierro a la posibilidad de que nuestro autor haya podido beber en alguna fuente antigua (trasladándola materialmente o «remodelándola»), pero que, tomando en consideración el alcance de mi investigación, no me queda más remedio que admitir que la «irrupción» conceptual y terminológica de García de Diego en lo que, desde Flydal y Coseriu, sin entrar en matices ahora, se denomina «eje diastrático» fue una entrada verdaderamente novedosa, por no decir revolucionaria en cuanto a la insistencia, concentración y terminología y que ha llegado el momento de que alguien se lance a una investigación de conjunto (diferentes ámbitos lingüísticos) para confirmar o no esta y otras realidades, sin descartar la posibilidad de que la terminología tempranamente utilizada por García de Diego haya trascendido al exterior y haya servido de inspiración (señalando o no la fuente) a algún estudioso no hispanohablante. Por lo que respecta a la aportación de Coseriu en este campo, vale la pena proseguir en la ruta que inicié en el volumen 7/1991 de esta revista.

$\mathrm{D} / 9$

Tras la breve entrega actual, puente entre el microsistema de referencias coserianas a los ejes diastrático y diafásico en algunas de sus lecturas y el microsistema de atisbos y formulaciones en su propia obra, nos toca comenzar (próximo número) tal desfile, en el que seguiré, como he hecho en el inmediatamente anterior, un orden cronológico. La diferencia estará en que, conforme avance la presentación de los trabajos aludidos (el primero será uno de 1949), se irá intensificando el grado de explicitud en la aprehensión y formulación de los conceptos y la terminología de los susodichos ejes diastrático y diafásico.

(continuará) 